\title{
A Saúde Mental dos Enfermeiros de Centro Cirúrgico: uma revisão integrativa
}

\section{Mental Health of Surgical Center Nurses: an integrative review}

\author{
Salud Mental de las Enfermeras de los Centros Quirúrgicos: una revisión integradora
}

Recebido: 10/07/2021 | Revisado: 15/07/2021 | Aceito: 17/07/2021 | Publicado: 25/07/2021

\author{
Vagner Marins Barcelos \\ ORCID: https://orcid.org/0000-0002-2826-1996 \\ Centro Universitário Anhanguera de Niterói, Brasil \\ E-mail: vagnerbarcelos@hotmail.com \\ Ana Cristina da Silva Oliveira \\ ORCID: https://orcid.org/0000-0002-8414-2561 \\ Centro Universitário Anhanguera de Niterói, Brasil \\ E-mail: ana.coliveira@educadores.net.br \\ Enéas Rangel Teixeira \\ ORCID: https://orcid.org/0000-0002-1721-2056 \\ Universidade Federal Fluminense, Brasil \\ E-mail: eneaspsi@hotmail.com \\ Pedro Paulo Corrêa Santana \\ ORCID: http://orcid.org/0000-0002-4470-9746 \\ Centro Universitário Anhanguera de Niterói, Brasil \\ E-mail: psantana.uff@gmail.com
}

\begin{abstract}
Resumo
Objetivo: identificar na literatura científica os fatores que impactam na saúde mental dos enfermeiros que atuam em centros cirúrgicos. Método: revisão integrativa da literatura com buscas nas bases de dados Literatura Latino Americana em Ciências da Saúde (LILACS), Medical Literature Analysis and Retrieval System Online (MEDLINE), Science Direct, Scopus e na Base de Dados em Enfermagem (BDENF). As buscas foram realizadas durante o mês de maio de 2021, referente ao período de janeiro de 2018 a maio de 2021. Foram considerados estudos disponibilizados integralmente, com conteúdo disponível gratuito e nos idiomas: inglês, português e espanhol. Resultados: foram obtidos 172 artigos do refinamento das buscas a partir da combinação dos descritores. Após retirada dos 31 estudos duplicados, 141 estudos seguiram para leitura dos resumos e títulos e foram selecionados 22 estudos para leitura integral e crítica. Foram selecionados três estudos para compor a revisão e, da análise temática, emergiu a categoria estressores no ambiente do centro cirúrgico. Conclusão: os fatores apontados como impactantes na saúde mental dos enfermeiros perioperatórios se relacionaram a fatores do ambiente de trabalho como a má relação interdisciplinar entre profissionais, a organização, a estrutura e o suporte aos enfermeiros. O estudo limitou-se a buscas de estudos publicados nos últimos três anos, o que pode ter relação com a escassez de publicações sobre a temática, inclusive publicações nacionais, representando a necessidade de mais estudos em saúde mental da enfermagem perioperatória.
\end{abstract}

Palavras-chave: Saúde Mental; Centros Cirúrgicos; Enfermeiras; Enfermagem.

\begin{abstract}
Objective: to identify the scientific literature and factors that impact the mental health of nurses who attend surgery centers. Method: integrative review of literature searching for databases on Latin American Literature in Health Sciences (LILACS), Medical Literature Analysis and Retrieval System Online (MEDLINE), Science Direct, Scopus and on Database on Sickness (BDENF). The studies were carried out during May 2021, referring to the period from January 2018 to May 2021. The studies were fully accomplished, and their content is available in Portuguese, English and Spanish. Results: 172 items have been obtained from search refinement and combination of descriptors. After removing 31 duplicated studies, 141 were selected as well as two abstracts and other titles; amongst them, 22 were chosen for comprehensive and critical reading. Three studies were elected to compose the review of the thematic analysis that emerged in the category: stressors in the environment of the surgical center. Conclusion: the factors identified as having an impact on the mental health of perioperative nurses were related to factors in the work environment, such as the poor interdisciplinary relationship between professionals, the organization, the structure and support for nurses. The study was limited to searches of studies published in the last three years, which may be related to the scarcity of publications on the subject, including national publications, representing the need for more studies on the topic of mental health in perioperative nursing.
\end{abstract}

Keywords: Mental Health; Surgicenters; Nurses; Nursing. 


\begin{abstract}
Resumen
Objetivo: identificar en la literatura científica los fatores que impactan la salud mental de los enfermeros que actúan en centros quirúrgicos. Método: revisión integrativa da literatura con búsquedas en las bases de dados Literatura Latino Americana en Ciências de la Salud (LILACS), Medical Literature Analysis and Retrieval System Online (MEDLINE), Science Direct, Scopus y en la Base de Dados en Enfermería (BDENF). Las búsquedas fueram hechas en mayo de 2021, referentes al periodo entre enero de 2018 y mayo de 2021. Se han seleccionados estudios disponibles en su totalidad, con contenido gratuito en portugués, inglés y español. Resultados: se obtuvieron 172 artículos de refinamiento de búsquedas a partir de la combinación de los descriptores; después retirada de los 31 estudios duplicados, 141 estudios seguidos para leer los resúmenes y títulos y se seleccionaron 22 estudios para la lectura completa y crítica. Se seleccionaron tres estudios para el examen y, del análisis temático, surgió la categoría: factores de estrés en el entorno del centro quirúrgico. Conclusión: Los factores identificados como de impacto en la salud mental de las enfermeras perioperatorias se relacionaron con factores del entorno laboral, como la mala relación interdisciplinaria entre los profesionales, la organización, estructura y apoyo de las enfermeras. El estudio se limitó a búsquedas de estudios publicados en los últimos tres años, lo que puede estar relacionado con la escasez de publicaciones sobre el tema, incluidas publicaciones nacionales, lo que representa la necesidad de más estudios sobre el tema salud mental de la enfermería perioperatoria.
\end{abstract}

Palabras clave: Salud Mental; Centros Quirúrgicos; Enfermeras; Enfermería.

\title{
1. Introdução
}

A saúde mental dos profissionais de saúde é uma temática que vem sendo ampliada como efeito das altas demandas originadas pelo serviço de alta complexidade. Considerando o trabalho um determinante para a saúde dos indivíduos em relação ao estilo de vida que apresentam, destaca-se a potencialidade quanto aos riscos psicossociais aos quais estão expostos. Sendo primordial destacar que os riscos psicossociais compreendem aqueles que depõem contra a saúde do trabalhador e desencadeiam adoecimento e comprometimento da qualidade do serviço prestado. No geral, os riscos psicossociais estão relacionados à esfera organizacional dos serviços, ao estilo de gestão, ao sofrimento patogênico e aos danos físicos, psicológicos e até mesmo sociais (Madrid et al., 2020; Fassarella et al., 2020).

O enfermeiro é o profissional que está imbricado na prevenção de agravos, na promoção e na reabilitação da saúde da população, o que exige desempenho de alta complexidade, com possibilidades de incorrer em riscos psicossociais, em todos os locais de atuação. No cenário hospitalar, em especial nos centros cirúrgicos, esses profissionais encontram-se em ambientes de alto risco, pois sujeitam-se a práticas dinâmicas, complexas, interdisciplinares, imprevistas e imediatistas. Logo, estes profissionais, além de realizarem o cuidado, estão sob forte pressão e são constantemente exigidos quanto ao trabalho em equipe, no que se relaciona a atividades de gestão de pessoas, de fluxo de pacientes, de equipamentos, de instrumentos, de materiais, o que configura um risco psicossocial para a saúde destes trabalhadores. (Madrid et al., 2020; da Silva Martins et al., 2021).

O centro cirúrgico (CC) é uma unidade onde os profissionais convivem com aspectos que os desafiam diariamente, tais como: intervenções de alto risco; atendimento a trauma; e situações de urgência. Estes fatores, associados ao estresse cotidiano e às longas jornadas de trabalho, influenciam na segurança e no bem-estar dos profissionais. O estresse sobre a saúde do trabalhador vai além do trabalho, pois pode afetar a sua vida pessoal e social. O trabalhador que convive com o estresse está mais suscetível ao surgimento dos sintomas. Além dos riscos à saúde física, os trabalhadores que sofrem com a sobrecarga de trabalho ficam expostos a maior risco de desenvolver doenças psiquiátricas. As relações de abuso ocorrem geralmente quando alguma pessoa se sente superior à outra, um olhar fragmentado e verticalizado do indivíduo (Glanzner \& Hoffmann, 2019; Ramos et al., 2021).

A busca pela qualidade de vida e a percepção do indivíduo como ser biopsicossocial propiciaram uma maior preocupação com a saúde do trabalhador. Assim, as organizações passaram a priorizar melhores condições de trabalho, no intuito de garantir a integridade física e psicológica dos profissionais, visando um ambiente de trabalho mais salubre. O tempo de atuação profissional pode ser um fator de proteção para a qualidade de vida no trabalho quando a organização oferece subsídios, como melhores salários, salubridade e respeito, pois o profissional se sente útil e valorizado. Todavia, nos cenários em que o 
serviço não se preocupa com a saúde do trabalhador, o aumento do tempo de serviço só gera desgaste, estresse e adoecimento (Carvalho et al., 2018; Fraga et al., 2019).

\section{Metodologia}

O presente artigo propõe uma revisão integrativa da literatura, de abordagem qualitativa, cujo método está fundamentado na prática baseada em evidências, ou seja, uma estratégica metodológica de busca de amplo alcance que, por meio da avaliação crítica, apresenta a síntese das evidências relevantes de tema específico (Ceolin et al., 2017; Estrela, 2018). Para elaboração desta revisão seguiram-se seis etapas: 1) elaboração da questão norteadora; 2) busca ou amostragem na literatura; 3) coleta de dados; 4) análise crítica dos estudos incluídos; 5) discussão dos resultados e 6) síntese da revisão. (Ceolin et al., 2017; Ferreira et al., 2019). Para síntese dos principais dados dos estudos selecionados, confeccionou-se um instrumento contendo: autores, ano de publicação, periódico, tipo de estudo, título da publicação.

A questão norteadora foi definida como: "Quais fatores impactam na saúde mental do enfermeiro atuante nos Centros Cirúrgicos?”. As buscas foram realizadas nas bases de dados: Literatura Latino Americana em Ciências da Saúde (LILACS), Medical Literature Analysis and Retrieval System Online (MEDLINE), Science Direct, Scopus e na Base de Dados em Enfermagem (BDENF) de artigos publicados entre a 2019 a 2021, considerando os primeiros casos notificados de covid-19 na China e a declaração de estado de pandemia de Covid-19 a partir de fevereiro de 2020 , com mudanças no cenário e nas rotinas dos profissionais de saúde, em especial dos enfermeiros. As buscas ocorreram em maio de 2021. Para a busca, definiram-se os Descritores em Ciências da Saúde (DeCS) / Medical Subject Headings (Mesh), com utilização dos descritores: "Enfermagem", "Enfermagem Perioperatória", "Saúde Mental", "Saúde do Trabalhador", e "Centros Cirúrgicos". Os descritores foram combinados aplicando-se o booleano "AND”, desta forma realizando um refinamento para melhor qualificar a pesquisa, visto a vasta literatura apresentada. As estratégias de buscas utilizadas nas bases de dados foram: 1) ("Enfermagem Perioperatória") AND (“Saúde do trabalhador”) AND (“Saúde Mental”); e 2) (“Enfermagem”) AND (“Centro Cirúrgico") AND (“Saúde Mental").

A seleção dos estudos para coleta de dados obedeceu aos critérios de inclusão: estudos de abordagem qualitativa e quantitativa, reflexões, estudos teóricos indexados em bases de dados, que versassem sobre a temática em questão, e que respondessem à pergunta de revisão, delimitando-se a estudos em língua portuguesa e língua inglesa, com disponibilização integral de conteúdo, de forma gratuita e publicados no período compreendido entre primeiro de janeiro de 2018 e 10 de maio de 2021. A publicação foi definida no período que coincide com o lançamento da campanha Nursing Now. Foram excluídos: editoriais, teses, dissertações, trabalhos de conclusão de curso, guias e manuais, e anais de eventos.

\section{Resultados}

Foram obtidos 172 estudos a partir das buscas nas bases de dados. Após a remoção dos duplicados, chegou-se ao total de 141 estudos que foram analisados inicialmente com leitura de títulos e resumos, sendo selecionados 22 estudos para leitura integral e análise crítica. Após análise dos estudos previamente selecionados, foram excluídos 19, sendo um por ser um editorial e não atender aos critérios de inclusão, e 18 por apresentarem outros pacientes ou outros profissionais de saúde que não eram enfermeiros perioperatórios. Assim, três estudos atenderam aos critérios de inclusão, versavam sobre a temática de saúde mental de enfermeiros atuantes em centros cirúrgicos e, também, respondiam à questão de revisão (Figura 1). 
Figura1 - Fluxograma do processo de identificação e seleção de artigos.

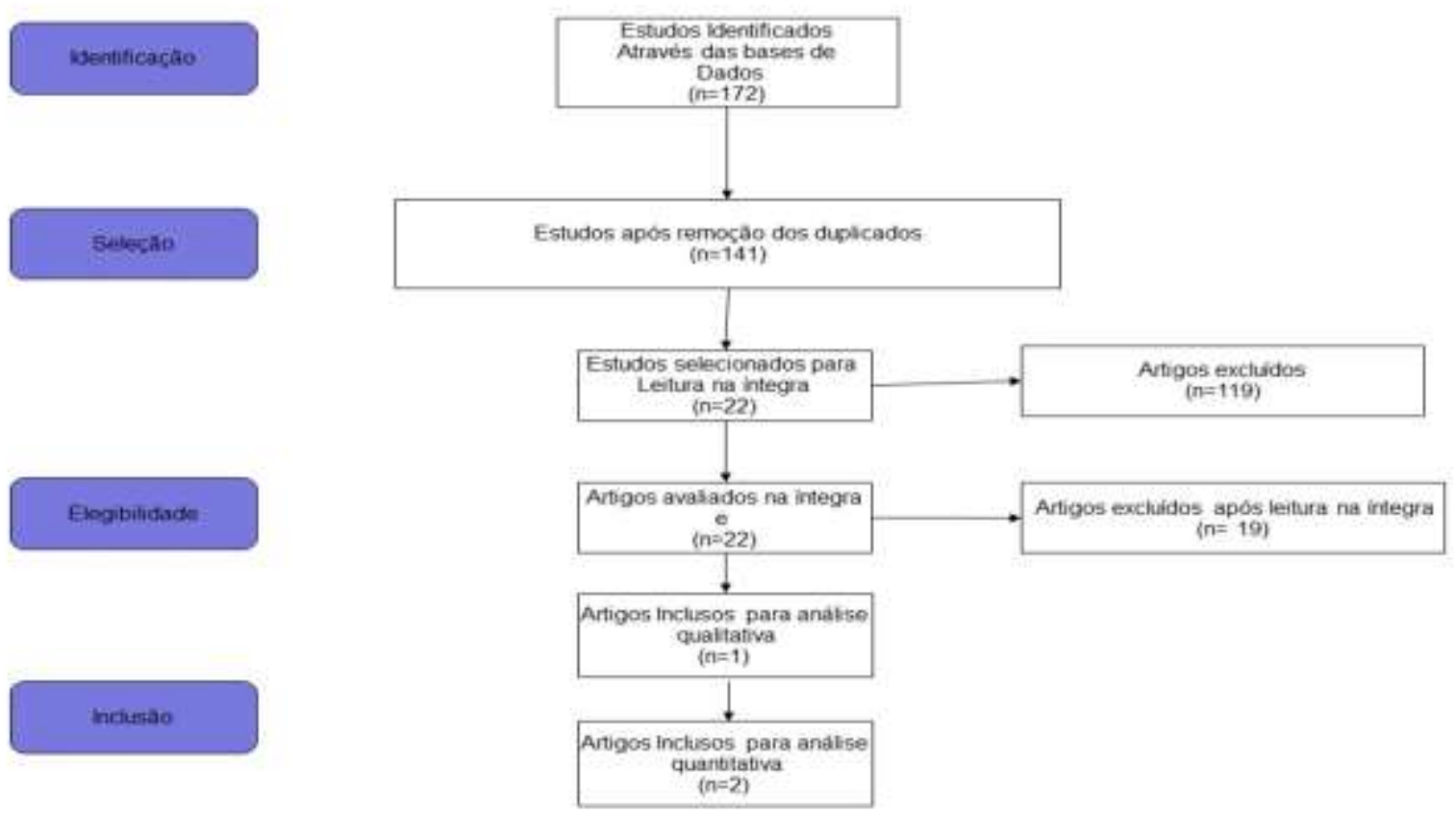

Fonte: Autores (2021).

Dos três estudos selecionados para compor a revisão, dois foram publicados em língua inglesa, e um publicado em língua portuguesa. Sobre a abordagem metodológica, dois estudos apresentaram abordagem metodológica de natureza quantitativa e um era da natureza qualitativa. Sobre o ano de publicação, dois foram publicados no ano de 2020 e um em 2018 (Quadro 1).

Quadro 1 - Caracterização dos estudos incluídos na revisão segundo autor, ano da publicação, idioma, país, objetivo, método e resultados.

\begin{tabular}{|c|c|c|c|c|c|}
\hline & $\begin{array}{l}\text { Identificação do } \\
\text { Artigo }\end{array}$ & Autoria & $\begin{array}{c}\text { Ano de } \\
\text { Publicação }\end{array}$ & Idioma & $\begin{array}{l}\text { País onde foi } \\
\text { conduzido o } \\
\text { estudo }\end{array}$ \\
\hline & E-01 & $\begin{array}{r}\text { Sillero, } \\
\text { A; Zabalegui, A. }\end{array}$ & 2018 & Inglês & Espanha \\
\hline Objetivo: & \multicolumn{5}{|c|}{$\begin{array}{l}\text { Determinar a influência dos fatores organizacionais do ambiente de trabalho do enfermeiro } \\
\text { perioperatório nas três dimensões do burnout: exaustão emocional, despersonalização e realização pessoal. }\end{array}$} \\
\hline Método: & \multicolumn{5}{|c|}{$\begin{array}{l}\text { Estudo transversal realizado com } 136 \text { enfermeiras de uma unidade de cuidados perioperatórios de } \\
\text { um hospital universitário de Barcelona, Espanha. Os dados foram coletados por meio de um formulário de } \\
\text { dados demográficos e as versões em espanhol da Escala de Ambiente de Prática do Trabalho de Enfermagem } \\
\text { e do Índice e Inventário de Burnout de Maslach. }\end{array}$} \\
\hline Resultados: & \multicolumn{5}{|c|}{$\begin{array}{l}\text { Os achados mostraram exaustão emocional em } 43 \% \text { (56) dos enfermeiros, despersonalização em } \\
21 \% \text { (28) e redução da realização pessoal em } 53 \% \text { (69). O grau de burnout geral foi moderado. O ambiente } \\
\text { de trabalho foi considerado desfavorável, pois apenas um fator de cinco era favorável (Fundamentos de } \\
\text { Enfermagem para uma Assistência de Qualidade). } \\
\text { As análises de regressão múltipla mostraram que três fatores organizacionais estavam associados a } \\
\text { todas as três dimensões do burnout: "capacidade de gestão da enfermeira, liderança e apoio dos enfermeiros", } \\
\text { "adequação de pessoal e recursos", e "fundamentos de enfermagem para uma assistência de qualidade". }\end{array}$} \\
\hline Conclusão & \multicolumn{5}{|c|}{ Neste estudo, três fatores organizacionais desempenharam um papel significativo na previsão de } \\
\hline
\end{tabular}




\begin{tabular}{|c|c|c|c|c|c|}
\hline & \multicolumn{5}{|c|}{$\begin{array}{l}\text { burnout entre os enfermeiros perioperatórios. Nós recomendamos que a gestão hospitalar implemente } \\
\text { políticas para melhorar esses fatores organizacionais. Promover estilos positivos de liderança, proporcionando } \\
\text { recursos necessários e a criação de um clima positivo no ambiente de trabalho pode aumentar o bem-estar } \\
\text { psicossocial e diminuir o burnout entre enfermeiras perioperatórias. }\end{array}$} \\
\hline & $\begin{array}{c}\text { Identificação do } \\
\text { Artigo }\end{array}$ & Autoria & $\begin{array}{c}\text { Ano de } \\
\text { Publicação }\end{array}$ & Idioma & $\begin{array}{l}\text { País onde foi } \\
\text { conduzido o } \\
\text { estudo }\end{array}$ \\
\hline & E-02 & $\begin{array}{r}\text { Sillero, } \\
\text { A; Zabalegui, A. }\end{array}$ & 2020 & Português & Espanha \\
\hline Objetivo: & \multicolumn{5}{|c|}{$\begin{array}{l}\text { Investigar como o ambiente laboral perioperatório afeta a insatisfação laboral, a exaustão profissional } \\
\text { e a percepção da qualidade do cuidado sobre a intenção de abandono do trabalho pelas enfermeiras } \\
\text { perioperatórias }\end{array}$} \\
\hline Método: & \multicolumn{5}{|c|}{$\begin{array}{l}\text { Estudo transversal com } 130 \text { enfermeiras que trabalham na área cirúrgica de um hospital universitário } \\
\text { público espanhol de alta tecnologia. Foram utilizados a escala do ambiente da prática de enfermagem, o } \\
\text { inventário de burnout de Maslach, as perguntas sobre a satisfação laboral, a percepção da qualidade do } \\
\text { cuidado e a intenção de abandonar o trabalho para a coleta de dados. Foram realizadas estatísticas descritivas, } \\
\text { inferenciais e de regressão logística. }\end{array}$} \\
\hline Resultados: & \multicolumn{5}{|c|}{$\begin{array}{l}20 \% \text { das enfermeiras perioperatórias gostariam de abandonar o trabalho. A dimensão do ambiente } \\
\text { laboral em relação aos investimentos em pessoal e recursos, a insatisfação e a exaustão emocional das } \\
\text { enfermeiras foram os fatores preditores que indicaram a sua intenção. }\end{array}$} \\
\hline \multirow[t]{3}{*}{ Conclusão } & \multicolumn{5}{|c|}{$\begin{array}{l}\text { Deve-se considerar implementar estratégias de retenção das enfermeiras perioperatórias, melhorando } \\
\text { os fatores preditores que indicam, como o ambiente laboral, especialmente a dotação de pessoal e os recursos, } \\
\text { a insatisfação e o esgotamento emocional. Criar ambientes de trabalho positivos, baseados nos valores } \\
\text { magnéticos, pode ser uma estratégia-chave. }\end{array}$} \\
\hline & $\begin{array}{c}\text { Identificação do } \\
\text { Artigo }\end{array}$ & Autoria & $\begin{array}{c}\text { Ano de } \\
\text { Publicação }\end{array}$ & Idioma & $\begin{array}{l}\text { País onde foi } \\
\text { conduzido o } \\
\text { estudo }\end{array}$ \\
\hline & E-03 & $\begin{array}{l}\text { Isik, I.; } \\
\text { Gümüskava, O; } \\
\text { Sem, S; Arslan, } \\
\text { Özkhan, H. }\end{array}$ & 2020 & Inglês & Turquia \\
\hline Objetivo: & \multicolumn{5}{|c|}{$\begin{array}{l}\text { Determinar as razões e as consequências das falhas na comunicação perioperatória e buscar } \\
\text { recomendações para melhoria. }\end{array}$} \\
\hline Método: & \multicolumn{5}{|c|}{$\begin{array}{l}\text { Estudo fenomenológico, realizado com entrevista com questionário semi-estruturado, seguindo as } \\
\text { sete etapas da metodologia Colazzi, tendo como participantes } 14 \text { enfermeiras perioperatórias. }\end{array}$} \\
\hline Resultados: & \multicolumn{5}{|c|}{$\begin{array}{l}\text { As categorias temáticas foram organizadas em: causas, consequências e recomendações para prevenir } \\
\text { falha de comunicação. Alguns temas para as causas eram tempo inadequado para o pré-operatório, preparação, } \\
\text { falta de pessoal e comportamentos perturbadores dos médicos. Consequências da falha de comunicação: } \\
\text { diminuição da retenção de pessoal, evitação de colegas, ameaças à segurança do paciente e violência dentro } \\
\text { da equipe. Das recomendações incluíram-se a aplicação de regulamentos institucionais e a criação de espírito } \\
\text { de equipe. }\end{array}$} \\
\hline Conclusão & \multicolumn{5}{|c|}{$\begin{array}{l}\text { O estudo revelou que os enfermeiros acreditam que as regulamentações institucionais não devem } \\
\text { apenas estar presentes, mas também aplicadas. Além disso, as enfermeiras acreditam que fortalecer as } \\
\text { habilidades interpessoais dos funcionários é essencial para prevenir problemas de comunicação. }\end{array}$} \\
\hline
\end{tabular}

Fonte: Autores (2021).

Emergiu da análise temática a seguinte categoria: estressores no centro cirúrgico. 


\section{Discussão}

A prática do enfermeiro atuante nos centros cirúrgicos é de extrema responsabilidade norteando fatores emocionais e organizacionais que podem incidir na saúde mental desses profissionais, ocasionando desconforto, mal-estar e até mesmo o sério acometimento profissional com a possibilidade de abandono da carreira.

\section{Estressores no Centro Cirúrgico}

No Estudo E-01 (Sillero \& Zabalegui, 2018), com 136 participantes, enfermeiros perioperatórios de um hospital universitário em Barcelona, Espanha os autores buscaram, através da coleta de dados com a utilização de dois questionários, um de dados demográficos e outro uma versão espanhola da Escala de Ambiente de Prática de Trabalho de Enfermagem, apresentar os fatores organizacionais e de esgotamento de enfermeiros perioperatórios segundo as três dimensões do esgotamento: exaustão emocional, despersonalização e realização pessoal. A partir dos dados, foram realizadas análises de correlação estatística que identificaram nível moderado-alto para exaustão emocional em 43\% (53) dos participantes, 21,5\% (28) para despersonalização e 53\% (69) para realização pessoal. Além disso, o estudo aponta para 41\% (53) dos participantes apresentaram alto nível de esgotamento, ou burnout, em termos gerais. Adiante, o estudo coloca que, na percepção dos participantes, o ambiente perioperatório foi considerado fator desfavorável àqueles nos escores médios, sendo estes referentes a: "participação do enfermeiro em assuntos hospitalares", "equipe e adequação de recursos", "habilidade da gerência de enfermagem, liderança e apoio dos enfermeiros", e "relações de colegas enfermeiros-médicos". 9

$\mathrm{O}$ achado mais relevante do estudo, segundo os autores, refere-se a três fatores organizacionais estarem associados ao esgotamento dos profissionais: "habilidade da gerência de enfermagem, liderança e apoio dos enfermeiros", "adequação de pessoal e recursos"; e "fundamentos de enfermagem para uma assistência de qualidade". O fator "habilidade da gerência de enfermagem, liderança e apoio dos enfermeiros" esteve associado à exaustão emocional e à despersonalização. Os autores ainda colocam que os resultados apontados representam a alta incidência do esgotamento, ou burnout, em enfermeiros na Espanha, e aponta para as necessidades de melhoria dos fatores organizacionais, estruturais, de suporte aos enfermeiros perioperatórios e das condições de trabalho, visando à segurança do paciente (Do Prado Tostes et al., 2017; Sillero \& Zabalegui, 2018).

No estudo E-02 (Sillero-Sillero \& Zabalegui, 2020), uma pesquisa transversal conduzida em um hospital universitário público de alta tecnologia na Espanha, com 130 enfermeiros perioperatórios participantes, os autores buscaram por meio da análise dos dados coletados, apresentar como o ambiente perioperatório de trabalho influencia na insatisfação com o serviço, na exaustão profissional e na percepção da qualidade do cuidado em relação à intenção de abandono do trabalho destes profissionais. O estudo teve como variáveis: o ambiente laboral, o nível de burnout, a percepção da qualidade do cuidado proporcionado, a intenção de abandonar o trabalho e a satisfação laboral. Em relação ao ambiente de trabalho, as participantes apresentaram como desfavoráveis: a habilidade, a liderança, o apoio dos responsáveis e a idoneidade do pessoal e recursos. Já em relação ao grau de exaustão, burnout, o estudo apontou que 6\% (n=5) enquadravam-se em nível alto e 47\% (n=36) apresentavam-se com nível médio. Além de 25\% ( $\mathrm{n}=19)$ apresentarem níveis altos de exaustão, $10 \%(\mathrm{n}=8)$ níveis altos de despersonalização e 21\% ( $\mathrm{n}=16)$ níveis altos de realização pessoal. No que se refere à satisfação laboral, o estudo aponta para 52\% dos participantes pouco satisfeitos, considerando que $20 \%$ tinham intenção de abandonar o emprego atual (Sillero-Sillero \& Zabalegui, 2020; Santana, 2018; Soares et al., 2017).

No estudo E-03 (Iş̧1k et al., 2020), estudo fenomenológico com 14 enfermeiros perioperatórios atuantes em dois hospitais em Istambul, Turquia, os autores buscaram compreender as razões e as consequências das falhas na comunicação. O estudo aponta que as falhas de comunicação estão relacionadas a conflitos na compreensão do papel dos enfermeiros, que sob o ponto de vista dos participantes, são tidos como secretários ou solucionadores de problemas.

Além disso, outro fator apontado como razão para a falha na comunicação foi o estresse advindo da pressão, uma vez 
que os participantes apontam que há pressão da equipe médica para terem pacientes preparados em tempos reduzidos, tornando o trabalho mais estressante, e com comportamentos de colegas com tratamento não adequado entre a equipe interdisciplinar, e a visão, pelos enfermeiros, de que são compreendidos como subordinados aos cirurgiões e não como co-trabalhadores. Adicionalmente, o estudo aponta as falas dos participantes que demonstraram já ter sofrido violência emocional, verbal e até mesmo física de integrantes da equipe cirúrgica, o que corrobora para o estresse e falhas na comunicação (Işsk et al., 2020; Dos Santos et al., 2019).

Os três estudos apontam para a realidade da enfermagem perioperatória considerando as condições de trabalho exaustivas, situações de relacionamento fragilizadas com outros profissionais de saúde da equipe, bem como as médias e altas taxas de burnout e a inclinação para o abandono do trabalho. Estes achados são compatíveis com a literatura científica e evidenciam os fatores que impactam na saúde mental dos enfermeiros perioperatórios. Além disso, evidenciam a necessidade de se fomentar um ambiente de trabalho amistoso, com estruturas e lideranças além de recursos compatíveis com a demanda laboral (Brito \& Pinheiro, 2017; Ribeiro, 2018; Munhoz et al., 2020). É importante salientar que não foram encontrados, nas buscas em bases de dados, estudos brasileiros sobre a saúde mental de enfermeiros perioperatórios.

\section{Conclusão}

Os fatores apontados como impactantes na saúde mental dos enfermeiros perioperatórios se relacionaram aos aspectos do ambiente de trabalho, como a má relação interdisciplinar entre profissionais, a organização, a estrutura e o suporte dos enfermeiros, sugerindo a realização de outras pesquisas. Além disso, os estudos evidenciaram grau médio e elevado de enfermeiros participantes com comprometimento de saúde tal como burnout em níveis variados, sendo mais proeminentes a despersonalização e a exaustão, fatos que representam a necessidade do cuidado com esses profissionais. O estudo limitou-se a buscas de estudos publicados nos últimos três anos, o que pode ter relação com a escassez de publicações sobre a temática, inclusive publicações nacionais, representando a necessidade de mais estudos sobre a saúde mental na enfermagem perioperatória.

\section{Referências}

Brito, C. F., \& Pinheiro, L. M. G. (2017). Caracterização do desconforto físico relacionado à ergonomia em profissionais de enfermagem do centro cirúrgico. Revista Enfermagem Contemporânea, 6(1), 20-29.

Carvalho, A. D. M. B., Cardoso, J. A., da Silva, F. A. A., Lira, J. A. C., \& Carvalho, S. M. (2018). Qualidade de vida no trabalho da equipe de enfermagem do centro cirúrgico. Enfermagem em foco, 9(3).

Ceolin, S., González, J. S., Ruiz, M. D. C. S., \& Heck, R. M. (2017). Bases teóricas de pensamento crítico na enfermagem ibero-americana: revisão integrativa da literatura. Texto \& Contexto-Enfermagem, 26. https://dx.doi.org/10.1590/0104-07072017003830016

da Silva Martins, J., Casarin, R. G., Schaffner, M. L., Fernandes, B. A. B., Machado, G. V., \& da Silva, L. A. A. (2021). Gestão de enfermagem no centro cirúrgico em hospital filantrópico, frente à pandemia COVID-19. Revista Ciência \& Humanização do Hospital de Clínicas de Passo Fundo, 1(1), 52-61.

do Prado Tostes, M. F., da Silva, A. Q., Garçon, T. L., Maran, E., \& Teston, E. F. (2017). Dualidade entre satisfação e sofrimento no trabalho da equipe de enfermagem em centro cirúrgico. Revista SOBECC, 22(1), 3-9.

dos Santos, D. A. C., Morais, D. S. V. D., Franco, R. V. B., \& Gomes, J. R. D. A. A. (2019). Qualidade de vida sob a ótica de enfermeiros do centro cirúrgico de um hospital público. Enfermagem em Foco, 10(4).

Estrela, C. (2018). Metodologia Científica: Ciência, Ensino, Pesquisa. Editora Artes Médicas.

Fassarella, C. S., Fernandes, L. F. G., da Silva Cavalcanti, R., Camerini, F. G., de Oliveira Meneses, R., \& de Souza, R. M. (2020). Cultura de segurança em centro cirúrgico universitário. Research, Society and Development, 9(8), e119985164-e119985164.

Ferreira, L., Barbosa, J. S. D. A., Esposti, C. D. D., \& Cruz, M. M. D. (2019). Educação Permanente em Saúde na atenção primária: uma revisão integrativa da literatura. Saúde em Debate, 43, 223-239.

Fraga, M. S. R., Calvetti, P. Ü., \& Lazzarotto, A. R. (2019). A qualidade de vida dos profissionais da enfermagem que atuam no centro cirúrgico. Barbarói, 1(53), 251-260. http://dx.doi.org/10.17058/barbaroi.v1i53.12986 
Research, Society and Development, v. 10, n. 9, e27710918091, 2021

(CC BY 4.0) | ISSN 2525-3409 | DOI: http://dx.doi.org/10.33448/rsd-v10i9.18091

Glanzner, C. H., \& Hoffmann, D. A. (2019). Fatores que interferem na saúde do trabalhador de enfermagem do centro cirúrgico: revisão integrativa. Revista Cubana de Enfermería, 35(4).

Işık, I., Gümüşkaya, O., Şen, S., \& Arslan Özkan, H. (2020). The Elephant in the Room: Nurses' Views of Communication Failure and Recommendations for Improvement in Perioperative Care. AORN journal, 111(1), e1-e15. https://doi.org/10.1002/aorn.12899

Madrid, B. P., Kotekewis, K., \& Glanzner, C. H. (2020). Trabalho da enfermagem no centro cirúrgico e os riscos psicossociais relacionados aos modos de gestão. Revista Gaúcha de Enfermagem, 41.

Munhoz, O. L., Andolhe, R., de Souza Magnago, T. S. B., Arrial, T. S., \& Moreira, L. P. (2020). Fatores associados ao estresse da equipe de enfermagem de unidade de clínica cirúrgica. Saúde (Santa Maria), 46(2). doi:https://doi.org/10.5902/2236583441499

Ramos, C. S., dos Santos, I. A. S., Silva, A. G. I., Barros, B. T. D., Barros, R. L. M., Bailão, R. O., ... \& Couto, A. M. F. A. (2021). Estresse ocupacional presente nas atividades da equipe de enfermagem em centro cirúrgico: Revisão integrativa. Research, Society and Development, 10(4), e13310413872-e13310413872. https://doi.org/10.33448/rsd-v10i4.13872

Ribeiro, V. F. (2018). Estresse e burnout entre enfermeiros perioperatórios. Revista SOBECC, 23(2), 59-60.

Santana, R. D. S. (2018). Percepções da equipe de enfermagem sobre acidentes com materiais perfurocortantes em um centro cirúrgico. Brazilian Journal of Surgery and Clinical Research-BJSCR, 24(3), 60-65.

Sillero, A., \& Zabalegui, A. (2018). Organizational factors and burnout of perioperative nurses. Clinical practice and epidemiology in mental health: CP \& $E M H, 14,132$. https://doi.org/10.2174/1745017901814010132

Sillero-Sillero, A., \& Zabalegui, A. (2020). Análise do ambiente laboral e intenção de enfermeiras perioperatórias de abandonar o trabalho. Revista LatinoAmericana de Enfermagem, 28. https://doi.org/10.1590/1518-8345.3239.3256

Soares, L. M. P., Oliveira, V. C., \& Sousa, L. A. A. (2017). Qualidade de vida dos profissionais atuantes no Centro Cirúrgico. Psicologia e Saúde em debate, $3(2), 159-170$. 